\title{
Predictors of physical activity in older adults experiencing an emergency hospital admission: a prospective cohort study
}

Peter Hartley ( $\nabla$ ph492@medschl.cam.ac.uk)

Cambridge University Hospitals NHS Foundation Trust https://orcid.org/0000-0002-1033-5897

Amanda L Dewitt

Cambridge University Hospital NHS Foundation Trust

Faye Forsyth

University of Cambridge Department of Public Health and Primary Care

Roman Romero-Ortuno

University of Dublin Trinity College

Christi Deaton

University of Cambridge Department of Public Health and Primary Care

Research article

Keywords: Aged, Hospital, Physical Activity, Accelerometers, Functional Mobility, Illness severity

Posted Date: February 4th, 2020

DOI: https://doi.org/10.21203/rs.2.22581/v1

License: (9) This work is licensed under a Creative Commons Attribution 4.0 International License.

Read Full License 


\section{Abstract}

\section{Background}

Reduced mobility may be responsible for functional decline and acute sarcopenia in older hospitalised patients. The drivers of reduced in-hospital mobility are poorly understood, especially during the early phase of acute hospitalisation. We investigated predictors of in-hospital activity during the first 24 hours of hospital admission in older adults.

\section{Methods}

This was a secondary analysis of a prospective repeated measures cohort study. Participants aged 75 years or older were recruited within the first 24 hours of admission. At recruitment, patients underwent a baseline assessment including measurements of pre-morbid functional mobility, cognition, frailty, falls efficacy, co-morbidity, acute illness severity, knee extension strength and grip strength, and consented to wear accelerometers to measure physical activity during the first 7 days (or until discharge if earlier). Inhospital physical activity was defined as the amount of upright time (standing or walking). To examine the predictors of physical activity, we used a best subset analysis including all baseline measures. The optimal model was defined by having the lowest Bayesian information criterion in the best-subset analyses. The model specified a maximum of 5 covariates and used an exhaustive search.

\section{Results}

Seventy participants were recruited but eight were excluded from the final analysis due to lack of accelerometer data within the first 24 hours after recruitment. Patients spent a median of 0.50 hours (IQR: $0.21 ; 1.43)$ standing or walking. The optimal model selected the following covariates: functional mobility as measured by the de Morton Mobility Index and two measures of illness severity, the National Early Warning Score, and serum C-reactive protein.

\section{Conclusions}

Physical activity, particularly in the acute phase of hospitalisation, is very low in older adults. The association between illness severity and physical activity may be explained by symptoms of acute illness being barriers to activity. Interdisciplinary approaches are required to identify early mobilisation opportunities.

\section{Background}

Unnecessarily reduced physical activity during hospitalisation may be part responsible for hospitalassociated functional decline and acute sarcopenia in older patients [1, 2]. Higher levels of physical activity whilst in hospital have been shown to correlate negatively with risk of death [3], length of stay [4], and functional decline [5], and positively with likelihood of discharge home [6]. 
Reports of the amount of physical activity in hospitalised older adults have varied, with median time spent standing or walking per day in hospital reported as being between 45 and 83 minutes [7-9]. Although this is considered 'low' it is unclear what the optimal level of activity is, and there are no universally accepted clinical guidelines in this area [10].

There has been an increase in clinical [11] and research focus $[6,12,13]$ to increase mobilisation on acute medical and geriatric wards, with an emphasis on protocols designed to improve the early and regular implementation of physical mobility activities to improve the health outcomes of hospitalised older people [14].

Previous studies have found pre-morbid levels of functional mobility $[7,9,10,15]$, cognitive ability [7] and acute illness severity [10] to be important determinants of ambulation in hospitalised older adults. However, the drivers of reduced in-hospital mobility are poorly understood, especially during the early phase of acute hospitalisation. We investigated clinical predictors of in-hospital activity during the first 24 hours of hospital admission in older adults.

\section{Method}

\section{Setting}

Patients were recruited from Cambridge University Hospital NHS Foundation Trust (CUH), a large tertiary university hospital in England with over 1000 beds. In 2018 CUH had 158,399 visits to the emergency department (ED), with 44,120 emergency admissions [16].

\section{Study Design}

This was a secondary analysis of a prospective repeated measures cohort study [17]. Ethical approval was granted by the London Queen Square Research Ethics Committee (17/LO/1817). All participants provided written informed consent.

\section{Patient and Public Involvement (PPI)}

Before the study began, a PPI panel was convened. The study design reflects amendments and changes suggested by the panel and patients. The panel also reviewed the final versions of the participant information sheet and consent form.

\section{Sample}

We included patients admitted to CUH over an 11-month period (Jan 2018 to Dec 2018), who were aged 75 years or older, experiencing an unplanned hospitalisation (i.e. non-elective), able to give informed consent and expected to be hospitalised for at least 48 hours. Exclusion criteria: admitted more than 24 hours before recruitment; unable to provide informed consent; receiving end-of-life care or treatment for diagnosed cancer; inability to cooperate in muscle-strength testing (e.g. unable to sit in chair, or skin 
integrity problem contraindicating the use of a hand-held dynamometer); transferred to or from the intensive care unit; bed-bound or requiring a hoist to transfer from bed to chair within the 2 weeks before hospitalisation; allergy to adhesive dressings; or if the clinical team had any other concerns regarding skin integrity around the proposed accelerometer sites. Sampling was convenience-based in that most screening took place Monday to Friday, 8:00 to 18:00. On recruitment days all patients over the age of 75 and admitted within the last 24 hours were consecutively screened by a member of the clinical team. Patients who met the inclusion criteria were approached regarding participation.

\section{Sample size}

The sample size was based pragmatically on maximising the number of recruits over an 11-month period.

\section{Procedures}

Participants were recruited during the first 24 hours of their hospital admission. At recruitment, a series of baseline measurements were taken, and the participants were fitted with accelerometers to measure physical activity.

\section{Measurements}

All measurements were taken by an experienced physiotherapist. Baseline measurements consisted of: age, sex, weight, frailty, acute illness severity, co-morbidity burden, falls efficacy, cognition, a self-reported measure of functional ability, a measure of functional mobility and objective physical activity levels via accelerometery.

The objective level of in-hospital physical activity was recorded using wearable accelerometers (AX3, Activity, Newcastle upon Tyne, UK), mounted mid-thigh and at the ankle, attached with adhesive dressings [18]. Data collected included the amount of time in a lying position, sitting position, standing position and walking [5]. The accelerometers were worn by participants after they provided informed consent, and were removed on day 7 or discharge, whichever was earliest.

The Survey of Health, Ageing and Retirement in Europe Frailty Instrument (SHARE-FI) tool was used to measure frailty. The SHARE-FI tool is a well validated and simple measurement of physical frailty [19]. Five SHARE variables approximating Fried's frailty phenotype definition are used: fatigue, loss of appetite, grip strength, functional difficulties and physical activity. Scores range between -2.7 and 13.4 (with 13.4 indicating the most severely frail) [19]. As it is routinely measured as part of clinical care, the Clinical Frailty Scale (CFS) score was also recorded [20]. The scoring of the CFS is based on a global assessment of patients' comorbidity symptoms, cognition, level of physical activity and dependency on activities of daily living. The possible scores range from 1 (very fit) to 9 (terminally ill).

To measure acute illness severity, we used serum C-reactive protein (CRP) levels, and the National Early Warning Score (NEWS), both of which are routinely collected on admission. CRP is an acute phase- 
reactant protein released in response to injury, infection or inflammation and is a recognised clinical measure of illness severity $[9,10]$. This was collected only for clinical reasons, therefore if CRP was not measured on admission but on day 1 of the study, then the day 1 value was used. The half-life of serum CRP in humans is approximately 19 hours $[11,12]$.

The NEWS was devised by the Royal College of Physicians of London to standardise the assessment and response to acute illness [21] and has been extensively validated [22].

The Charlson Comorbidity Index (CCl) is a method for classifying comorbid conditions for use as a prognostic indicator [23]. The $\mathrm{CCl}$ is based on patients' diagnoses as coded by the World Health Organization's International Classification of Diseases $\left(10^{\text {th }}\right.$ version).

Falls efficacy is defined as self-perceived confidence in engaging in activities of daily living without falling [24]. The FES-I is a reliable and validated measure in older adults [25].

The Mini-ACE is a 30-point scale used to detect cognitive impairment [26]. The Mini-ACE has been reported to have higher sensitivity and higher ceiling effect than the Mini-Mental State Examination [26] . A score of 25 or less is suggestive of cognitive impairment [26] .

Self-reported general functional ability was measured using the Barthel Index: a 10-item ordinal scale (0100) of functional independence with activities of daily living, where a score of 100 represents a high level of functional independence [27]. The participants were asked at baseline assessment to base their answers on their functional ability two weeks before admission.

The DEMMI is a 100-point ordinal scale for the assessment of mobility in older acute medical patients [28]. It consists of 15 items ranging from assessing bed mobility to high levels of dynamic balance. A score of 100 represents a high level of functional mobility [28]. The DEMMI provides interval level measurement and does not have floor or ceiling effects in the acute hospital setting.

In-hospital physical activity was defined as the amount of upright time (standing or walking).

Analysis

Data were analysed with R software [29]. Continuous variables were described as median or inter-quartile range (IQR), and categorical variables as count and percentage. The analysis was limited to the first 24 hours of measured activity due to attrition of data as patients were discharged from hospital.

To examine the predictors of physical activity in the first 24 hours of study participation we used a best subset analysis using the 'leaps' R package [30]. To comply with the assumption of normality of residuals, upright time was transformed using a base-10 logarithm transformation. The model specified a maximum of 5 covariates and used an exhaustive search. To assess for overfitting of the data, the Bayesian information criterion (BIC) was extracted. We also performed k-fold cross validation $(k=10)$ to predict the different models' ability to generalise to independent data sets. 


\section{Results}

Seventy participants were recruited but eight were excluded from the final analysis due to lack of accelerometer data within the first 24 hours. Reasons for missing data included: clinical need for MRI scans and therefore unable to wear accelerometers $(n=3)$, withdrawal $(n=3)$, contraindicating skin condition $(n=1)$, and device malfunction $(n=1)$. A further 15 patients only wore one accelerometer on their thigh due to poor skin condition on their lower legs. For these participants it is not possible to differentiate between lying and sitting. Participant characteristics and levels of activity are presented in Table 1. There was a median time of 19.6 hours (IQR: $15.9 ; 22.9)$ between admission to hospital and baseline assessment.

The results of the best subset analysis are presented in Table 2; all variables in Table 1 were included in the analysis. According to the BIC, the optimal model for predicting physical activity level on admission was model 3, which used functional mobility (DEMMI) and two measures of illness severity (NEWS and CRP) as the co-variates (see Table 3). Further increasing the number of co-variates only marginally increased the adjusted $\mathrm{R}^{2}$ value. Figure 1 illustrates the relationship between the back-transformed sedentary activity and the three independent variables. The DEMMI is plotted as a continuous variable on the $x$-axis, the NEWS and CRP are depicted at 3 arbitrary levels along their continuous scales, the NEWS score 2,4 and 6 as separate lines, and CRP level $0 \mathrm{~m} / \mathrm{L}, 100 \mathrm{~m} / \mathrm{L}$ and $200 \mathrm{~m} / \mathrm{L}$ as separate facets.

Table 1. Participant Characteristics $(n=62)$ 


\section{Characteristics}

Female

Age (years)

Weight $(\mathrm{kg})$

CFS

SHARE-FI

CCI

Admission CRP (mg/L)

NEWS

Barthel Index (self-reported 2-weeks prior to admission)

DEMMI

FES-I
Summary measure: median (IQR) or count (\%)

$26(41.9 \%)$

$85.0(80.2 ; 87.0)$

$68.7(56.2 ; 78.8)$

$4.5(4.0 ; 5.0)$

$3.4(1.6 ; 4.3)$

$2.0(1.0 ; 3.0)$

$46.0(8.1 ; 119.2)$

$2.5(1.0 ; 4.0)$

$90.0(76.2 ; 100.0)$

$41.0(33.8 ; 56.0)$

$26.5(20.2 ; 33.8)$ 
Mini ACE

Grip strength $(\mathrm{kg})$

Knee torque $(\mathrm{Nm})$

Active time (standing or walking) first 24 hours of study (hours)
$27.0(25.0 ; 28.8)$

$18.2(14.0 ; 23.6)$

$43.4(33.8 ; 57.8)$

$0.50(0.21 ; 1.43)$

Abbreviations: CFS= Clinical Frailty Scale; SHARE-FI= Survey of Health, Ageing and Retirement in Europe Frailty Instrument; $\mathrm{CCI}=$ Charlson Comorbidity Index; CRP= C-reactive protein; NEWS= National Early Warning Score; DEMMI= de Morton Mobility Index; FES-I= Falls Efficacy Scale - International; Mini-ACE= Mini-Addenbrooke's Cognitive Assessment.

Table 2. Results of best subset analysis 


\begin{tabular}{|l|l|l|l|l|}
\hline Model & Covariates in model & BIC & Adjusted $\mathbf{R}^{2}$ & Cross-validation error \\
\hline 1 & DEMMI & -4.65 & 0.18 & 0.15 \\
\hline 2 & DEMMI, NEWS & -8.67 & 0.27 & 0.14 \\
\hline 3 & DEMMI, NEWS, CRP & -12.61 & $\mathbf{0 . 3 5}$ & $\mathbf{0 . 1 3}$ \\
\hline 4 & DEMMI, NEWS, CRP, CCI & -10.98 & 0.36 & 0.14 \\
\hline 5 & DEMMI, NEWS, CRP, CCI, Sex & -8.26 & 0.37 & 0.14 \\
\hline
\end{tabular}

Abbreviations: $\mathrm{BIC}=$ Bayesian information criterion, $\mathrm{DEMMI}=$ de Morton Mobility Index; NEWS= National Early Warning Score; $\mathrm{CRP}=\mathrm{C}$-reactive protein; $\mathrm{CCl}=$ Charlson Comorbidity Index

Table 3. Summary of model 3 from best subset analysis

\begin{tabular}{|l|l|l|l|l|}
\hline Term & $\begin{array}{l}\text { Estimate (base 10 logarithmic } \\
\text { transformation) }\end{array}$ & $\begin{array}{l}\text { Lower 95\% } \\
\text { CI }\end{array}$ & $\begin{array}{l}\text { Upper 95\% } \\
\text { CI }\end{array}$ & $\begin{array}{l}\text { P } \\
\text { value }\end{array}$ \\
\hline DEMMI & 0.01 & 0 & 0.01 & $<.001 *$ \\
\hline NEWS & -0.03 & -0.04 & -0.01 & $.002 *$ \\
\hline CRP & 0.00 & 0.00 & 0.00 & $.007 *$ \\
\hline
\end{tabular}


Abbreviations: DEMMI= de Morton Mobility Index; NEWS= National Early Warning Score; $C R P=C-$ reactive protein

\section{Discussion}

In this study, patients were active for only 30 minutes during the first twenty-four hours after recruitment (median time from hospital admission to recruitment was 19.6 hours). This study confirms findings from previous studies which indicate a low proportion of time spent being active in the early period following an acute admission [7-9, 15].

The best subset model found functional mobility and illness severity to be most predictive of physical activity during the initial period of hospitalisation, explaining $35 \%$ of variance in activity time. Higher levels of functional mobility ability and lower illness severity (lower admission CRP and NEWS) were associated with higher amounts of physical activity. These findings are in keeping with previous research regarding the predictive value of functional mobility $[7,9,10,15]$, and illness severity $[10]$ in hospitalised older people.

The association between illness severity and physical activity may be explained by symptoms of acute illness being barriers to activity. Previous studies have highlighted concerns around mobilising patients who are acutely ill, such as respiratory and cardiovascular instability, or who have indwelling devices, weakness, pain, polypharmacy, sleep disturbance, and reduced nutritional intake [31-33].

Functional mobility scored by the DEMMI is likely to reflect the amount of assistance a person needs in order to mobilise. For example, a person with a score of 38/100 would be expected to be able to perform bed based mobility tasks, require minimal assistance or supervision for transfers in and out of the chair, have adequate balance to sit and stand unsupported and walk short distances with assistance or supervision [28]. The availability of staff to provide assistance is frequently cited as a barrier to activity in hospital [31-33]. The functional mobility scores may also reflect clinicians' assessments of risk of falls, which may affect clinicians' behaviour and advice, resulting in reduced patient activity [32]. Indeed, $11 \%$ of the cohort described by C So and E Pierluissi [31] reported being actively discouraged from walking, although whether this was to do with a perceived risk of falling, illness severity or another reason is unclear.

It has been hypothesised that older adults with low falls efficacy may restrict their daily activities [34]. Qualitative research has highlighted fear of falling as a barrier to activity in hospital [31, 32]. Fall efficacy however, was not identified as a top predictor of physical activity in any of the 5 models, nor was their evidence of multicollinearity (indicating that falls efficacy was accurately predicted by functional mobility and illness severity).

In the absence of guidelines regarding optimal levels of physical activity in older hospitalised patients, we 
are unable to determine whether any participants regardless of functional ability or illness severity were adequately active. However, particularly for the least active patients, this work may challenge the belief held by some that it is the patient's lack of motivation that limits physical activity in hospital [32]. To increase activity in the least active patients, it is hypothesised in the context of these findings, that more staff able to provide physical assistance and appropriately risk assess the dangers of mobilising or indeed not mobilising acutely sick patients are needed. This approach requires an interdisciplinary collaboration between medical, nursing and therapy personnel, similar to the approach that has been reported in intensive care settings [35].

\section{Limitations}

There are several limitations to this research including the small sample size, and the use of a convenience sampling method. Patients with significant cognitive impairments were excluded from this study for reasons of informed consent. Cognitive impairment has been shown to be common in hospitalised older adults and is associated with in hospital functional decline [36] and reduced activity [7].

This is also a secondary data analysis, which therefore limits the potential variables explored regarding this specific research question. There is also no qualitative data included in the analysis, as none was collected.

\section{Conclusions}

Physical activity, particularly in the acute phase of hospitalisation, is very low in older adults. Of the variables included in this study, functional mobility and illness severity were found to be the best predictors of physical activity in this acute phase. Given these findings to increase physical activity in the least active patients, it is predicted that more staff trained to provide physical assistance and able to risk assess mobility appropriately in acutely ill patients are needed.

\section{List Of Abbreviations}

BIC: Bayesian information criterion

CCl: Charlson Comorbidity Index

CFS: Clinical Frailty Scale

CUH: Cambridge University Hospital NHS Foundation Trust

CRP: C-reactive protein

DEMMI: de Morton Mobility Index 
DME: Department of Medicine for the Elderly

ED: Emergency Department

FES-I: Falls Efficacy Scale - International

IQR: Inter-quartile range

Mini-ACE: Mini-Addenbrooke's Cognitive Assessment

NEWS: National Early Warning Score

PPI: Patient and Public Involvement

SHARE-FI: Survey of Health, Ageing and Retirement in Europe Frailty Instrument

\section{Declarations}

\section{Ethics approval and consent to participate}

Ethical approval was granted by the London Queen Square Research Ethics Committee (17/LO/1817). All participants provided written informed consent.

\section{Consent for publication}

Not applicable

\section{Availability of data and materials}

The datasets generated and/or analysed during the current study are not publicly available as permission was not gained for this from our participants but are available from the corresponding author on reasonable request.

\section{Competing interests}

The authors declare that they have no competing interests

\section{Funding}

Peter Hartley is funded by a research training fellowship from The Dunhill Medical Trust [grant number RTF115/0117]. Additional funding for the study was provided by The Addenbrooke's Charitable Trust [grant reference: 32/17 A (ii)]. Roman Romero-Ortuno is funded by Science Foundation Ireland [grant number 18/FRL/6188].

\section{Authors' contributions}


$\mathrm{PH}$ : concept and design, acquisition of subjects, data collection, analysis, interpretation and manuscript preparation. AD: Concept for the paper, interpretation of the data, manuscript preparation. FF:

interpretation of the data, manuscript preparation. RRO: design of study, interpretation of the data, manuscript preparation. CD: design of study, interpretation of the data, manuscript preparation. All authors read and approved the final manuscript.

\section{Acknowledgements}

We thank all the members of the Acute Medicine and DME wards in our hospital and all the participants for making this study possible.

\section{References}

1. Lafont $C$, Gérard S, Voisin T, Pahor M, Vellas B: Reducing 'iatrogenic disability' in the hospitalized frail elderly. Journal of Nutrition, Health \& Aging 2011, 15(8):645-660 616p.

2. Welch C, Z KH-S, C AG, J ML, T AJ: Acute Sarcopenia Secondary to Hospitalisation - An Emerging Condition Affecting Older Adults. Aging Dis 2018, 9(1):151-164.

3. Brown CJ, Friedkin RJ, Inouye SK: Prevalence and outcomes of low mobility in hospitalized older patients. J Am Geriatr Soc 2004, 52(8):1263-1270.

4. McCullagh R, Dillon C, Dahly D, Horgan NF, Timmons S: Walking in hospital is associated with a shorter length of stay in older medical inpatients. Physiol Meas 2016, 37(10):1872-1884.

5. Agmon M, Zisberg A, Gil E, Rand D, Gur-Yaish N, Azriel M: Association Between 900 Steps a Day and Functional Decline in Older Hospitalized Patients. JAMA Intern Med 2016.

6. Liu B, Moore JE, Almaawiy U, Chan WH, Khan S, Ewusie J, Hamid JS, Straus SE, Collaboration MO: Outcomes of Mobilisation of Vulnerable Elders in Ontario (MOVE ON): a multisite interrupted time series evaluation of an implementation intervention to increase patient mobilisation. Age Ageing 2018, 47(1):112-119.

7. Pedersen MM, Bodilsen AC, Petersen J, Beyer N, Andersen O, Lawson-Smith L, Kehlet H, Bandholm T: Twenty-four-hour mobility during acute hospitalization in older medical patients. $J$ Gerontol A Biol Sci Med Sci 2013, 68(3):331-337.

8. Brown CJ, Redden DT, Flood KL, Allman RM: The underrecognized epidemic of low mobility during hospitalization of older adults. J Am Geriatr Soc 2009, 57(9):1660-1665.

9. Villumsen M, Jorgensen MG, Andreasen J, Rathleff MS, Molgaard CM: Very Low Levels of Physical Activity in Older Patients During Hospitalization at an Acute Geriatric Ward: A Prospective Cohort Study. J Aging Phys Act 2015, 23(4):542-549.

10. Fisher SR, Graham JE, Brown CJ, Galloway RV, Ottenbacher KJ, Allman RM, Ostir GV: Factors that differentiate level of ambulation in hospitalised older adults. Age Ageing 2012, 41(1):107-111.

11. Oliver D: David Oliver: Fighting pyjama paralysis in hospital wards. BMJ 2017, 357:j2096. 
12. Brown CJ, Foley KT, Lowman JD, Jr., MacLennan PA, Razjouyan J, Najafi B, Locher J, Allman RM: Comparison of Posthospitalization Function and Community Mobility in Hospital Mobility Program and Usual Care Patients: A Randomized Clinical Trial. JAMA Intern Med 2016, 176(7):921-927.

13. Lim S, Ibrahim K, Dodds R, Purkis A, Baxter M, Rogers A, Sayer AA, Roberts HC: Physical activity in hospitalised older people: the feasibility and acceptability of a volunteer-led mobility intervention in the SoMoVe study. Age Ageing 2019.

14. Smart DA, Dermody G, Coronado ME, Wilson M: Mobility Programs for the Hospitalized Older Adult: A Scoping Review. Gerontol Geriatr Med 2018, 4:2333721418808146.

15. Evensen S, Sletvold O, Lydersen S, Taraldsen K: Physical activity among hospitalized older adults - an observational study. BMC Geriatr 2017, 17(1):110.

16. A\&E Attendances and Emergency Admissions [https://www.england.nhs.uk/statistics/statisticalwork-areas/ae-waiting-times-and-activity/]

17. Hartley P, Romero-Ortuno R, Wellwood I, Deaton C: Changes in Muscle Strength and Physical Function in Older Patients During and After Hospitalisation: a Prospective Repeated-Measures Cohort Study. Age \& Ageing 2019, Submitted.

18. Hartley P, Keevil VL, Westgate K, White T, Brage S, Romero-Ortuno R, Deaton C: Using Accelerometers to Measure Physical Activity in Older Patients Admitted to Hospital. Curr Gerontol Geriatr Res 2018, 2018:3280240.

19. Romero-Ortuno R, Walsh CD, Lawlor BA, Kenny RA: A Frailty Instrument for primary care: findings from the Survey of Health, Ageing and Retirement in Europe (SHARE). BMC Geriatrics 2010, 10(1):112.

20. Rockwood K, Song X, MacKnight C, Bergman H, Hogan DB, McDowell I, Mitnitski A: A global clinical measure of fitness and frailty in elderly people. CMAJ 2005, 173(5):489-495.

21. Royal College of Physicians of London: National early warning score (NEWS): standardising the assessment of acute-illness severity in the NHS-report of a working party. In.; 2012.

22. Pimentel MAF, Redfern OC, Gerry S, Collins GS, Malycha J, Prytherch D, Schmidt PE, Smith GB, Watkinson PJ: A comparison of the ability of the National Early Warning Score and the National Early Warning Score 2 to identify patients at risk of in-hospital mortality: A multi-centre database study. Resuscitation 2019, 134:147-156.

23. Charlson ME, Pompei P, Ales KL, MacKenzie CR: A new method of classifying prognostic comorbidity in longitudinal studies: development and validation. J Chronic Dis 1987, 40(5):373-383.

24. Tinetti ME, Richman D, Powell L: Falls efficacy as a measure of fear of falling. J Geronto/1990, 45(6):P239-243.

25. Yardley L, Beyer N, Hauer K, Kempen G, Piot-Ziegler C, Todd C: Development and initial validation of the Falls Efficacy Scale-International (FES-I). Age Ageing 2005, 34(6):614-619.

26. Hsieh S, McGrory S, Leslie F, Dawson K, Ahmed S, Butler CR, Rowe JB, Mioshi E, Hodges JR: The Mini-Addenbrooke's Cognitive Examination: a new assessment tool for dementia. Dement Geriatr Cogn Disord 2015, 39(1-2):1-11. 
27. Mahoney Fl, Barthel DW: Functional Evaluation: The Barthel Index. Md State Med J 1965, 14:61-65.

28. de Morton NA, Davidson M, Keating JL: The de Morton Mobility Index (DEMMI): an essential health index for an ageing world. Health Qual Life Outcomes 2008, 6:63.

29. R Core Team: R: A Language and Environment for Statistical Computing. R Foundation for Statistical Computing. In., 3.5.1 edn. Vienna, Austria; 2018.

30. Lumley T based on Fortran code by Miller A: leaps: Regression Subset Selection. R package version 3.0. https://CRAN.R-project.org/package=leaps. 2017.

31. So C, Pierluissi E: Attitudes and expectations regarding exercise in the hospital of hospitalized older adults: a qualitative study. J Am Geriatr Soc 2012, 60(4):713-718.

32. Brown CJ, Williams BR, Woodby LL, Davis LL, Allman RM: Barriers to mobility during hospitalization from the perspectives of older patients and their nurses and physicians. J Hosp Med 2007, 2(5):305313.

33. Hoyer EH, Brotman DJ, Chan KS, Needham DM: Barriers to early mobility of hospitalized general medicine patients: survey development and results. Am J Phys Med Rehabil 2015, 94(4):304-312.

34. Hughes CC, Kneebone, II, Jones F, Brady B: A theoretical and empirical review of psychological factors associated with falls-related psychological concerns in community-dwelling older people. Int Psychogeriatr 2015, 27(7):1071-1087.

35. Dafoe S, Chapman MJ, Edwards S, Stiller K: Overcoming barriers to the mobilisation of patients in an intensive care unit. Anaesth Intensive Care 2015, 43(6):719-727.

36. Hartley P, Gibbins N, Saunders A, Alexander K, Conroy E, Dixon R, Lang J, Luckett J, Luddington T, Romero-Ortuno R: The association between cognitive impairment and functional outcome in hospitalised older patients: a systematic review and meta-analysis. Age Ageing 2017, 46(4):559-567.

\section{Figures}



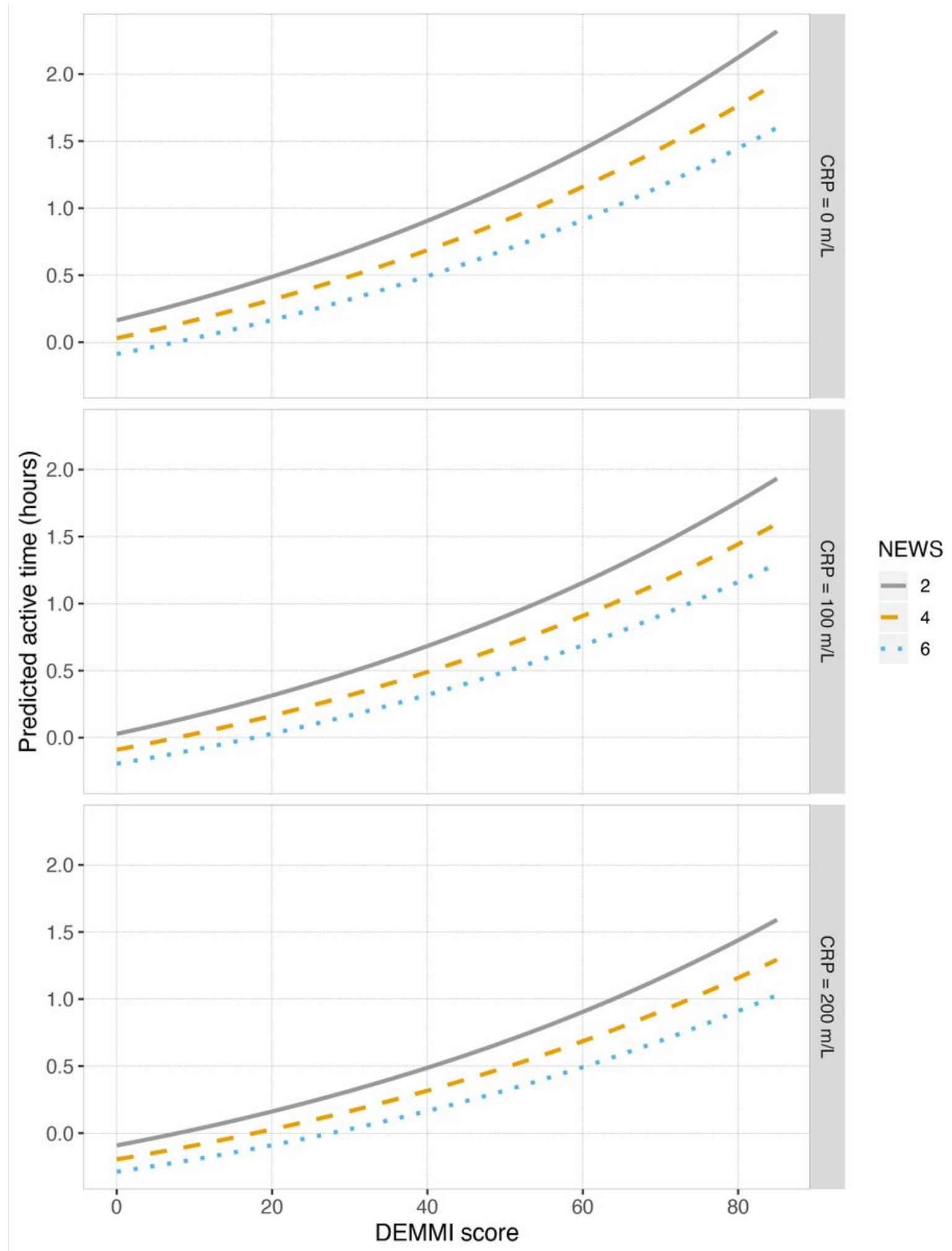

\section{Figure 1}

Active time (hours) as predicted by functional mobility and illness severity (back transformed regression) Abbreviations: $\mathrm{CRP}=$ C-reactive protein; $\mathrm{DEMMI}=$ de Morton Mobility Index; NEWS = National Early Warning Score 